\title{
DIPOLE COUPLING EFFECT OF HOLOGRAPHIC FERMION IN CHARGED DILATONIC GRAVITY
}

\author{
WEN-YU WEN \\ Department of Physics, Chung Yuan Christian University, \\ Chung Li City, Taiwan \\ wenw@cycu.edu.tw \\ SHANG-YU WU \\ Institute of Physics, National Chiao Tung University, \\ Hsinchu 300, Taiwan \\ loganwu@gmail.com
}

\begin{abstract}
In this note, we study the dipole coupling effect of holographic fermion in a charged dilatonic black hole proposed by Gubser and Rocha. ${ }^{1}$ It is found that the property of Fermi liquid is rigid under perturbation of dipole coupling, and the Fermi momentum is linearly shifted. A gap is dynamically generated as the coupling becomes large enough and the Fermi surface ceases to exist as the bulk dipole coupling further increases.
\end{abstract}

Keywords: AdS-CFT correspondence.

\section{Introduction and Summary}

A boundary theory dual to the AdS Reissner-Nordström black hole in the presence of a dipole interaction term is proposed $^{2}$ and studied in several following works: Refs. 3-8. Upon tuning the dipole coupling strength, the boundary theory is found in a phase of either Fermi liquid, non-Fermi liquid or Mott insulator. We remark that while the extremal AdS RN background, without dipole coupling, was first claimed to be dual to a boundary theory of non-Fermi liquid at quantum critical point, ${ }^{10}$ it is not clear which role does the dipole coupling play in a boundary theory of Fermi liquid. It is the goal of this paper to explicitly investigate the dipole coupling effect on a particular boundary theory of Fermi liquid, ${ }^{a}$ which is dual to the charged dilatonic black hole proposed by Gubser and Rocha. ${ }^{1}$

Here, we summarize our finding as follows:

- We have found the structure of Fermi surface constructed by Gubser and Rocha is rigid regardless of the presence of dipole coupling. There is no sign about the Fermi/non-Fermi liquid transition by varying bulk dipole coupling.

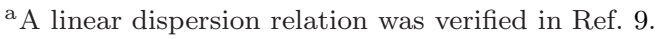


- Fermi momentum can be linearly shifted by the dipole coupling strength.

- A gap appears for large dipole coupling strength, as a generic feature of dipole interaction. However, the gap might not be the Mott one, since we could not observe any clear sign of spectral weight transfer from the spectral function.

- The gap persists at finite temperature for large enough dipole coupling.

\section{Rigidity of Fermi Surface}

Now we would like to introduce the dipole coupling in the charged dilatonic black hole proposed by Gubser and Rocha. ${ }^{1}$ Let us consider the bulk spinor action,

$$
\mathcal{S}_{D}=i \int d^{4} x \sqrt{-g} \bar{\psi}(\not D-m-i p \not \mathbf{F}) \psi,
$$

We found that the dipole coupling does not change the structure of Fermi surface but just shift the location of Fermi momentum. The gradually disappearance of Fermi surface with increasing $p$ at the metal-insulator transition agrees with the ARPES measurement in some strongly-correlated oxides such as $\mathrm{La}_{1-x} \mathrm{Sr}_{x} \mathrm{MnO}_{3} .{ }^{11}$

We do not observe any sign of non-Fermi liquid numerically by tuning the dipole coupling strength $p$. The linear relation between $k-k_{F}$ and $\omega$ implies the Fermi surface constructed in Ref. 1 is rigid and the property of Fermi liquid remains regardless of the bulk dipole coupling. The group velocity $v_{g}=\partial \omega / \partial k$ can also be read off as $v_{g} \simeq 0.79 c$ for $p \leq 1$ and slows down for larger $p$, say $v_{g} \simeq 0.5 c$ for $p=5$. We can see the pole of Green function is shifted linearly up to some value of $p$, before the whole system enters the insulator phase where the pole disappears. The numerical result shows the best fit for relation: $k-k_{0} \propto 0.66103 p^{1.00161}$, where $k_{0}=2.19213747$ is the numerically-found Fermi momentum at $p=0$.

\section{Acknowledgments}

This work is supported by the Taiwan's National Science Council and the NCTS.

\section{References}

1. S. S. Gubser and F. D. Rocha, "Peculiar properties of a charged dilatonic black hole in $\mathrm{AdS}_{5}, "$ Phys. Rev. D 81, 046001 (2010) [arXiv:0911.2898 [hep-th]].

2. M. Edalati, R. G. Leigh and P. W. Phillips, Phys. Rev. Lett. 106, 091602 (2011).

3. M. Edalati, R. G. Leigh, K. W. Lo and P. W. Phillips, Phys. Rev. D 83, 046012 (2011).

4. D. Guarrera and J. McGreevy, arXiv:1102.3908 [hep-th].

5. J. P. Gauntlett, J. Sonner and D. Waldram, Phys. Rev. Lett. 107, 241601 (2011).

6. J. Gauntlett, J. Sonner and D. Waldram, JHEP 1111, 153 (2011).

7. W.-J. Li and H. Zhang, JHEP 1111, 018 (2011).

8. J. P. Wu and H. B. Zeng, arXiv:1201.2485 [hep-th].

9. J. P. Wu, Phys. Rev. D 84, 064008 (2011).

10. H. Liu, J. McGreevy and D. Vegh, Phys. Rev. D 83, 065029 (2011).

11. A. Chikamatsu, H. Wadati, H. Kumigashira, M. Oshima, A. Fujimori, M. Lippmaa, K. Ono, M. Kawasaki and H. Koinuma, Physical Review B 76, 201103 (2007). 